\title{
$\frac{100}{\mathrm{LuT}} \mathrm{RPES}$
}

KRZYSZTOF JAJUGA*

\section{THE DEVELOPMENT OF THE ECONOMIC SCIENCES: TWO CONTEMPORARY TENDENCIES}

\section{INTRODUCTION}

Each scientific discipline has its own characteristics: the way it has developed, the connections with the other disciplines, the prevailing methodology, and the way the research is conducted. This is also true for the economic sciences. This paper discusses two important trends shaping research in the economic sciences over the last two decades.

The first one is the growing interdisciplinarity of economic research. The second one is the impact of technological changes on research in the economic sciences. The paper presents the author's systematization of these problems. Section 2 provides a presentation of the economic sciences, understood as a discipline within the overall classification of scientific disciplines - with a particular emphasis on Polish regulations. Some other classifications of scientific disciplines are discussed in Section 3. The development of the economic sciences over the last few decades is discussed in Section 4. The first contemporary trend - growing interdisciplinarity in economic research - is presented in Section 5. A presentation of some technological innovations is provided in Section 6 . The second contemporary trend - the impact of technological changes on research in the economic sciences - is presented in Section 7.

\section{ECONOMIC SCIENCES AS A DISCIPLINE}

In this paper I follow the classical approach to the classification of scientific disciplines, in which two levels are distinguished. The first one is the

* Krzysztof Jajuga, Uniwersytet Ekonomiczny we Wrocławiu, krzysztof.jajuga@ue.wroc.pl, https://orcid.org/0000-0002-5624-6929. 
level of fields of science, in which wide areas are recognized, such as the humanities, social sciences, natural sciences, etc. The second one is the level of scientific disciplines, wherein a field of science contains a number of scientific disciplines.

The traditional approach that can be applied in understanding the economic sciences consisted in distinguishing three disciplines:

- Economics;

- Finance;

- Management science.

This approach was also to large extent adopted in Polish regulations. Scientific degrees in the field of economic sciences were awarded in the above three disciplines (plus a fourth one, commodity science, which contained research in the economic sciences, as well as in the agricultural sciences and chemical sciences). In the aforementioned regulations, the economic sciences were one of seven fields of science.

In 2018 the regulatory changes in the classification of scientific disciplines took place in Poland, resulting in three disciplines in the economic sciences being distinguished, namely: economics and finance, management and quality sciences, socio-economic geography and spatial economy. In addition, these three disciplines have become the part of the social sciences, rather than being separate field of science. These new regulations decreased the number of fields of science from 22 to 8 and the number of disciplines from 95 to 47 .

In this paper, I concentrate mainly on economics and finance, but for the sake of simplicity I use the term 'economic sciences'.

One of the most well-known classifications of scientific disciplines is the one established by the OECD. ${ }^{1}$ It was used as a benchmark for the classification used in new Polish regulations. The OECD proposed the following systematization within the field of the social sciences:

- Psychology;

- Economics and Business, including:

- Economics, Econometrics; Industrial relations; Business and Management;

- Educational sciences;

- Sociology;

- Law;

- Political science;

- Social and economic geography;

- Media and communications;

- Other social sciences.

There are two differences in Polish regulations compared to the OECD classification:

- canonical law as a separate discipline;

- economics and finance formally separated from business and management.

1 See <http://www.oecd.org/science/inno/38235147.pdf>. 


\section{OTHER CLASSIFICATIONS OF DISCIPLINES}

The above-mentioned classification given in the Polish regulations (and used by the Scientific Excellence Council) is applied almost solely for the purpose of awarding the following scientific title and degrees:

- title of professor awarded by the President of Poland;

- habilitation degree awarded by a university (or other scientific institution);

- doctoral degree awarded by a university (or other scientific institution).

However, it is interesting to look at the classifications that are applied when one wants to classify research in the economic sciences without explicit relation to titles and degrees. These classifications can be used:

- either formally, usually in the applications for research grants;

- or less formally, for example in assigning a paper submitted to a scientific journal to classes or subclasses.

I will discuss several such classifications, in which - in addition to the economic sciences - I will mention some related disciplines of social sciences. The following classifications will be discussed:

- one established by the NCN (National Science Centre);

- one established by the European Research Council;

- the Journal of Economic Literature's classification.

\section{The classification of the National Science Centre}

This classification was established for the purpose of the evaluation of research projects within relatively homogeneous groups. The economic sciences belong to the large class HS (Arts, Humanities and Social Sciences) and to the subclass HS4 called 'Individuals, institutions, markets'. This subclass contains the following disciplines: economics, finance, management, demography, social and economic geography, urban studies.

One can see that there are similarities to the classification used for scientific titles and degrees. The only exceptions are demography and urban studies, which are recognized as separate disciplines.

Further, on the lower level, sixteen subdisciplines are mentioned, namely:

- Macroeconomics;

- Microeconomics, institutional economics;

- Econometrics, statistical methods;

- Population dynamics, demographic processes;

- Resources and sustainable development;

- Financial markets, international finance, public finance;

- Banking, corporate finance, accounting;

- Behavioural economics, consumption and consumer behaviour, marketing;

- Organization studies, strategic management, concepts and methods of management, logistics;

- Human resource management, employment and salaries; 
- Public economics, social infrastructure, public administration;

- Living conditions and standards, income distribution, poverty;

- International economics;

- Human and social geography;

- Land management, urban studies;

- Other related subjects.

One can see that in this classification 11 subdisciplines belong to 'economics and finance', 2 disciplines belong to 'management and quality science', and the other 2 belong to 'socio-economic geography and spatial economy'.

\section{The classification of the European Research Council}

This classification was established by the European Research Council for the evaluation of research projects. In the area of Humanities and Social Sciences, there is a discipline 'Economics, finance and management', which is divided into the following subdisciplines:

- Macroeconomics.

- Development, economic growth;

- Microeconomics, behavioural economics;

- Marketing;

- Political economy, institutional economics, law and economics;

- Econometrics, statistical methods;

- Financial markets, asset prices, international finance;

- Banking, corporate finance, accounting;

- Competitiveness, innovation, research and development;

- Organization studies: theory and strategy, industrial organization;

- Labour economics, income distribution and poverty;

- Public economics;

- International trade;

- History of economic thought and quantitative economic history.

As could have been expected, the classification made by the NCN was based on the one made by the ERC.

\section{The classification of the Journal of Economic Literature}

This classification is now considered as a standard one for assigning scientific papers within the economic sciences. The Journal of Economic Literature is published by the American Economic Association and contains survey papers. This journal established the classification system which is now used by many journals in the field of the economic sciences. Authors assign their papers to respective classes and subclasses and state this in the paper, in addition to keywords.

This classification (updated quarterly) is a hierarchical one, containing three levels: the first one is a general category, denoted by letter, while the second and third categories, middle and basic, respectively, are both denoted by digits. 
The first level contains 20 general categories:

A. General Economics and Teaching.

B. History of Economic Thought, Methodology and Heterodox Approaches.

C. Mathematical and Quantitative Methods.

D. Microeconomics.

E. Macroeconomics and Monetary Economics.

F. International Economics.

G. Financial Economics.

H. Public Economics.

I. Health, Education and Welfare.

J. Labour and Demographic Economics.

K. Law and Economics.

L. Industrial Organization.

M. Business Administration and Business Economics. Marketing. Accounting. Personnel Economics.

N. Economic History.

O. Economic Development, Innovation, Technological Change and Growth.

P. Economic Systems.

Q. Agricultural and Natural Resource Economics. Environmental and Ecological Economics.

R. Urban, Rural, Regional, Real Estate and Transportation Economics.

Y. Miscellaneous Categories.

Z. Other Special Topics.

The total number of basic categories in this classification amounts to 856 (!).

This classification contains overlapping categories. For example, if we consider papers published within wide area of Finance, and search just for the term 'finance' in the JEL classification, we find:

- One general category $(\mathrm{G})$.

- Three middle categories (F3, F4, N2).

- 17 basic categories (B17, B27, C58, D14, D25, D53, E44, F65, I22, O16, P34, P43, P45, Q14, R51, Z22, Z33).

In addition, there are several basic categories containing terms closely related to finance, like investment, interest rates, budget, etc. Therefore, it is usually the case that the authors assign one paper to several basic categories.

This classification shows many relations of the economic sciences to other disciplines and even fields of science. It is worth mentioning such disciplines as: Psychology, Sociology, Law, Culture, Health, Education and Research, Sports, and Tourism.

This shows the interdisciplinarity of economic research. I will refer to this fact in Section V.

\section{Nobel Memorial Prize in Economic Sciences - different areas}

Another possible idea for the classification of the economic sciences may be derived from the analysis of the scientific achievements for which Nobel 
Memorial Prize in Economic Sciences was awarded. As is well known, this prize was established in 1968 and it is funded by central bank of Sweden (Riksbank). The first prize was awarded in 1969. Since that time 52 prizes have been awarded to 86 scientists.

The analysis of the scientific achievements for which the prize has been awarded leads to the recognizing the main areas within the economic sciences. However, one must remember that the prize is awarded for life achievements, which were often published even several dozen years ago, therefore this is a more retrospective analysis.

My analysis is based on the classification proposed by Assar Lindbeck, ${ }^{2}$ who conducted an analysis on the prizes awarded until 2007. I extended this analysis until 2020. Here is the list of areas and the names of Nobel Memorial Prize laureates:

Econometrics:

Macroeconometrics:

Macroeconomics:

Macroeconomics and Institutional Economics:

Microeconomics and Economic Sociology:

Microeconomics:

International Economics:

International Macroeconomics:

International and Regional Economics:

Development Economics:

Labour Economics:

Welfare Economics:

Financial Economics:

Economic History:

Administrative (Management) Science:

Economic Psychology and Experimental Economics:

Economics of Information:

Public Finance:

Industrial Organization:

Game Theory:

Auction Theory:

National Income Accounting:
Frisch, Tinbergen, Haavelmo, Heckman, McFadden, Engle, Granger

Klein, Sargent, Sims

Tobin, Modigliani, Lucas, Friedman, Phelps, Kydland, Prescott

Myrdal, von Hayek

Becker

Hurwicz, Maskin, Myerson

Ohlin, Meade

Mundell

Krugman

Schultz, Lewis

Diamond, Pissarides, Mortensen

Sen, Deaton, Banerjee, Duflo, Kremer

Markowitz, Sharpe, Miller, Merton, Scholes, Fama, Shiller, Hansen

Fogel, North

Simon

Kahneman, Smith, Thaler

Mirrlees, Vickrey, Akerlof, Stiglitz, Spence

Buchanan

Stigler, Tirole

Harsanyi, Nash, Selten, Aumann, Schelling, Roth, Shapley

Milgrom, Wilson

Stone

\footnotetext{
${ }^{2}$ Lindbeck (2008).
} 
General Equilibrium Theory:

Partial and General Equilibrium Theory:

Economic Growth Theory:

Economic Growth and Economic History:

Input-Output Analysis:

Theory of Optimal Allocation of Resources:

Theory of Institutions:

Economic Governance:

Contract Theory:
Hicks, Arrow, Debreu

Samuelson, Allais

Solow, Nordhaus, Romer

Kuznets

Leontief

Koopmans, Kantorowicz

Coase

Ostrom, Williamson

Hart, Holmstrom

The following conclusions can be drawn from this systematization:

- The areas in which the most prizes were awarded are: Macroeconomics, Econometrics and Finance.

- There are a few non-mainstream areas, which were recognized as worthy of awards, for example: Economics of Information, Auction Theory, Contract Theory.

- Apart from purely theoretical achievements, some prizes (especially in the last decade) have been awarded for achievements that are widely applied (or even practice-driven), for example: Game Theory, Auction Theory, Welfare Economics; here very often the arguments for the award are supported by practical usefulness.

- Some achievements have an interdisciplinary character, for example: Economic Psychology, Economic Governance.

\section{THE EVOLUTION OF THE ECONOMIC SCIENCES IN THE LAST DECADES}

The last decade of the twentieth century was characterized by significant changes in the world, particularly around economic systems. On the one hand, many countries, particularly in Central and Eastern Europe, began the process of transition from a centrally planned economy to the market economy (Poland being the leader of these changes). On the other hand, the process of globalization started. It had many driving forces, but one of them was the transition process that took place in many countries.

These changes led to higher economic growth (measured by growth of GDP per capita) in many countries, which also resulted in an increase of wealth in the majority of households. These facts had an impact on the research and findings in economic sciences. In some research it was indicated that one of the reasons for the economic success of many countries was the suitable economic policy, which was based on the classical theories.

One of the facts confirming this statement was successful monetary policy. In many countries the objective of this policy was to set a medium-term infla- 
tion target as a nominal anchor. This helped to cut the inflation rate in the countries which had begun the transition process.

Another important fact was the high economic growth in the first few years of the twenty-first century. We could observe the perception of many economists, who attributed the economic success to good economic theory, particularly based on classical monetary theory. This was the dominant perception in the first years of twenty-first century. This perception gradually changed in the period following Great Financial Crisis, which had its origins in the years 2006 and 2007 and then culminated in the years 2008 and 2009.

In addition, we observed growing criticism of the economic sciences, as well as of economists, who were accused of failing to forecast the crisis, and in fact they did not prevent the crisis. In my opinion, this criticism is not fully justified. Here are some arguments.

Firstly, the financial crisis in the United States was partially caused by political decisions encouraging banks to give loans to poor households (the so called sub-prime loans) to realize the vision of 'American dream'.

Secondly, very often the critical assessment of economists and the economic sciences was made by the representatives of other disciplines, who perhaps did not understand that the essence of economic phenomena is very complicated, since the development of these phenomena depends strongly on the individual and collective actions of humans. These actions are not driven by precise models (like, for example, many models in physics), but by humans whose behaviour very often cannot be predicted with a high level of accuracy.

Despite these arguments, it is clear that the financial crisis, followed by economic crisis, revealed the failure of some theories which had been treated as 'revealed truths'.

There are two main streams of research in the economic sciences. Normative economic sciences (or: normative economics) propose models which describe the desirable behaviour of economic agents (entities). Here the key assumption is the rationality of agents. Positive economic sciences (or: positive economics), sometimes also called descriptive economic sciences, analyse the actual behaviour of economic agents. The criticism of economic sciences which was voiced after the financial crisis, refers mostly to normative economics.

In my opinion, the main weaknesses of the economic sciences, particularly of their normative version, result from the following features:

1. Models derived in the economic sciences are often based on assumptions that are not justified by an analysis of real world. Sometimes such assumptions were made to get a much easier path to the final form of the model. A significant example of this was the use of the pricing model for credit derivatives based on the so-called Gaussian copula valuation. In fact, this assumption became completely invalid in the summer of 2007, and this caused the losses of the portfolios of some investment banks.

2. The main criticism referred to the assumption of the rational agent (entity, person, etc.), the so-called homo oeconomicus. Richard Thaler, the laure- 
ate of the Nobel Memorial Prize in economic sciences in 2017 uses the name 'econ' for rational agents, and claims that people are humans, not 'econs'.

3. Many models derived in economic sciences are not robust enough to cope with the changes in the economy, but these changes are very dynamic. Some models are not verified empirically, they will remain only in the theoretical sphere (for example, some models derived in mathematical economics). Other models are verified, but only using historical data. If, however, a model which was valid in the past is not robust enough to deal with changes in the economy, then this model might be not useful in the real world. Until recent years, many models have not been subject to 'what if' analysis. In finance, such analysis belongs to the analysis of model risk.

4. Theories derived in economic sciences are sometimes not created for solving real world problems. It even happened in the past that pure theoretical papers were evaluated more highly than empirical papers. In my opinion, the economic sciences should address problems which arise with human activities in real world. If this does not happen, the scientists conducting research in economic the sciences will still face criticism.

5. In the years preceding the financial crisis, mainstream economics dominated scientific research and didactics, while nonclassical streams were not so often considered.

6. It is worth mentioning that there are cases in which some practitioners have deeper and more updated knowledge than some theoreticians. This is particularly observed in the areas in which it is still too early to have conclusions coming from scientific research. One example is part of the financial markets research - this area is characterized by a great deal of innovation in the instruments and processes.

In my opinion, the economic sciences are facing the following dilemmas ${ }^{3}$ :

1. Normative versus positive economic sciences

Since normative economic sciences have often been criticized, it looks like we will see the growing importance of research in the positive economic sciences, particularly analysing the behaviour of economic agents. However, one should not neglect the normative economic research, because it should lead to recommendations about desirable behaviour. In addition, the role of normative theories in the teaching process is not be overvalued.

\section{The formalization of economic sciences}

The history of scientific achievements in the economic sciences, for example those for which the Nobel Memorial Prize in Economic Sciences were awarded, indicates that there has been high degree of formalization, particularly using mathematical tools. There is no doubt that some level of formalization is needed, to avoid lack of precision and logic in the research (some of them are called 'qualitative research' to hide the low methodological level). Some of this research results in papers containing merely subjective description and

\footnotetext{
3 See Jajuga (2019).
} 
deriving general conclusions, not based on thorough inference and not having the property of generalization.

On the other hand, sometimes formalization can lead to the introduction of unjustifiable and unrealistic assumptions. This is an element of the so-called model risk, which can be defined as the risk that the model is not useful for the real world. In my opinion, the important role of the economic sciences is firstly, explanation of the real world, secondly, providing solutions for practitioners. This means that a model should be robust enough to cope with the changes in the real world and be transparent for the end user.

3. Verification of theory versus exploration of data

The substantial part of the research in the economic sciences uses data (numerical or textual). There are two types of methods in which the data is used:

- Confirmatory methods, in which there is verification of existing theories with the use of available data;

- Exploratory methods, in which data analysis is used to discover some patterns that could form the basis of a new theory.

The use of exploratory methods has been growing in last two decades. There are three reasons for this trend:

- dynamic changes in real world led to new phenomena for which there is no available theory, therefore it is not possible to use confirmatory methods;

- there is an enormous growth of available data;

- technological progress allows more efficient (fast and precise) data analysis.

The last two features will be discussed in the next sections of this paper.

\section{INTERDISCIPLINARITY IN THE ECONOMIC SCIENCES: THE FIRST CONTEMPORARY TREND}

It was already mentioned in a previous section that research in economic sciences have been increasingly related to the other scientific disciplines. One good example is the award of the Nobel Memorial Prize in Economic Sciences for the research in behavioural economics (Daniel Kahneman, Richard Thaler). In my opinion, this trend will continue. There are at least two driving forces behind this tendency.

\section{Explanation of human actions through different lenses}

As I have mentioned above, the standard classification by the JEL covers some other disciplines that are related to the economic sciences. In my opinion, these are the disciplines which can provide deep explanations of the actions of economic agents (entities), particularly their behaviour. Among the numerous disciplines, I would mention the following: 


\subsection{Psychology}

This discipline studies the behaviour of humans, particularly behaviour in economic activities. The segment of this discipline related to economic sciences is called either 'behavioural economics' (respectively 'behavioural finance') or 'economic psychology'. One of the origins of behavioural economics comes from a paper by Kahneman and Tversky, ${ }^{4}$ which is one of the most cited papers in the economic sciences. The research papers in behavioural economics or behavioural finance often consider either cognitive biases (involved in the complicated matter of decision making) or emotional biases (due to the fact that humans are not necessarily rational agents, but they are driven by emotions or some subjective preferences).

\subsection{Sociology}

This discipline, when related to economic activities, studies, on the one hand, the social effects of economic phenomena, and on the other hand, the impact of social connections on the economic decision-making. The segment of this discipline related to economic sciences is called 'economic sociology' or 'socioeconomics' (or 'social finance'). The relations between sociology and economic sciences were analysed many years ago. One of the origins of contemporary economic sociology (the so-called 'new economic sociology') comes from a paper by Granovetter. ${ }^{5}$ In my opinion, there is new possible stream of research in economic sociology, resulting from the dynamic growth of social media activities and the huge impact of these activities (including the dissemination of fake news) on the behaviour of entities.

\subsection{Neurophysiology}

This discipline, when related to economic activities, studies the functioning of brain and its impact on economic decision-making. This type of research is usually conducted using imagery and biochemical tests, relating them to the process of economic decision-making. The outcome of such research can be findings about the impact of some parts of the brain on human decisions. The segment of this discipline related to economic sciences is called 'neuroeconomics' (or 'neurofinance'). One of the first papers in neuroeconomics was published by Platt and Glimcher. ${ }^{6}$

\subsection{Cultural Studies}

This discipline, when related to economic activities, studies the impact of different elements of culture on economic decision-making. These different ele-

\footnotetext{
${ }^{4}$ Kahneman, Tversky (1979).

5 Granovetter (1985).

6 Platt, Glimcher (1999).
} 
ments of culture that can be analysed are, for example: religion, tradition, arts, literature, philosophy, etc. The segment of this discipline related to economic sciences is called 'cultural economics' (or 'cultural finance'). One of the first papers in cultural finance was published by Guiso, Sapienza and Zingales. ${ }^{7}$

\subsection{Legal science}

This discipline, when related to economic activities, studies the impact of legal regulations on economic decision-making. Different jurisdictions have different impacts on economic systems. One of the most significant examples is the so-called Islamic finance, based on the legal rules derived from Islam. One of the most important connections between the economic sciences and legal science is the economic analysis of law, which consists in the application of economics in the analysis of legal regulations. It has origins in the papers by Coase $^{8}$ and Calabresi. ${ }^{9}$

These disciplines which are related to the economic sciences can be seen as a structure which aims at the explanation of human economic decision making. The main link can be derived from:

- external factors: legal rules and culture, as well as social relations, and

- internal factors: psychology determined through brain actions.

\section{The application of methods used in other disciplines in the economic sciences}

The relations between the economic sciences and the other disciplines are also developed on the basis of the methods used in the other disciplines. This mostly refers to the fact that quantitative research in economic sciences depends on the tools adopted from widely understood areas of mathematical sciences, by which I mean: econometrics, statistics, applied mathematics (calculus, linear algebra, probability).

In addition to interdisciplinarity, understood as the application of the theory and methods of the other disciplines in economic sciences, it is also worth mentioning the application of economic methodology in some other areas.

Economic research deals mostly with the actions of individuals (generally: entities) in the economy. These actions are explained by theories derived through scientific research. The question arises of whether the methods and theories used in economic sciences can be applied to explain other human activities, possibly some life activities.

It seems that the pioneer of this approach was Gary Becker, the Nobel Memorial Prize in economic sciences laureate in 1992. He proposed the use of classical microeconomic analysis in non-market activities, such as: investments in human capital, behaviour of the family, distribution of work and allocation of time in the family, crime and punishment.

\footnotetext{
7 Guiso, Sapienza, Zingales (2004).

8 Coase (1961).

9 Calabresi (1961).
} 
Other examples of the application of economic methodology are:

- economic analysis of law,

- economic research on such segments of the economy, as: health, education, culture, and sports.

\section{TECHNOLOGY: AN IMPORTANT FACTOR FOR CHANGES IN THE ECONOMY}

As has already been discussed, one of the main roles of the economic sciences is the analysis and explanation of economic phenomena (including, for example, financial markets). It is therefore worth looking at the important factors having a strong impact on the economy. The most important changes in the economy are driven by:

- Geopolitical situation.

- Social changes.

- Climate changes;

- Technological development;

- Some unexpected global events, like COVID-19.

In this paper I concentrate on the impact of technological development on the research in economic sciences. Before discussing this in more detail, it will be worth providing some systematized insights into main innovative technological changes. Here I consider only those changes that have a significant impact on the economic sciences, since they refer to the real economy and the financial markets.

In my opinion, these innovative technological changes can be systematized into two classes:

- 'hard' innovations (hardware);

- 'soft' innovations (software).

Among the 'hard' innovations I distinguish:

- Developments in computer technology, resulting in increases in the speed of computations;

- Developments in telecommunication technology, resulting in increases in the speed of data transmission;

- Cloud computing, resulting in the use of participation in common resources allowing data processing.

The effect in the first two areas can be seen by looking at some numbers.

The speed of computation is measured by the number of floating-point operations per second (so called FLOPS). In 1985 the fastest computer in the world, supercomputer Cray 2 , had a speed equal to 1.9 gigaflops $\left(10^{\wedge} 9\right.$ FLOPS). At present, the fastest computer in the world, Fugaku, has a speed equal to 415.5 petaflops $\left(10^{\wedge} 15\right.$ FLOPS), which is about 218 million times faster than Cray 2. The fastest processor used today in commercial smartphones, Qualcomm Snapdragon 865Plus, has a speed equal to 122,38 gigaflops, which is 64 times faster than Cray 2. The next progress in the speed of computation can be expected with the advancements of quantum computers. 
The speed of transmission is measured by two numbers: bandwidth and latency. The first one is the amount of data that depends purely on transmission technology. At present the maximal bandwidth is 178 TB per second. The second one, latency, means the time it takes to send data from one place to another. If we take as an example the distance from London to New York $(5570 \mathrm{~km})$, it takes light in vacuum about 19 milliseconds, and for the fastest optical fiber about 28 milliseconds, to travel this distance.

These facts mean that:

- on the one hand, all the data used in economic research can be processed much faster than previously, which can accelerate research,

- on the other hand, economic research can be conducted around these 'hard' innovations, by looking at economic costs and social benefits.

Among 'soft' innovations I distinguish:

- methods for the acquisition and analysis of data, including the so-called Big Data and methods of machine learning,

- advanced techniques used in roboadvisory and algorithmic trading in financial markets,

- the reporting of transactions and contracts in distributed ledger technology, including blockchain.

Out of these innovations, there are three of which - in my opinion - will have an impact on the way that research in the economic sciences is conducted. I will briefly introduce them.

\section{Big Data}

Big Data are large, changing and heterogeneous data sets, for which processing, and analysis is difficult, however this can add value if it leads to knowledge acquisition. The dynamic growth of Big Data has been possible because of the growth of terminals connected through the internet. Until 2003, humankind produced about 5 exabytes $\left(10^{\wedge} 15\right.$ bytes) of data, now this amount of data is produced in less than 45 minutes.

Big Data contains heterogeneous sets of data. The classical data are numerical data, containing value of continuous and discrete data, presented on nominal, ordinal, interval and ratio scales. In addition, nowadays also textual data are considered as classical data. But there has been enormous growth of the so-called alternative data, including video files, audio files, satellite images, sensory data, etc.

One of the oldest concepts to use alternative data in economic sciences was the proposal of Henderson, Storeygard and Weil ${ }^{10}$ to use a proxy when GDP growth is measured poorly or not measured at all, for towns or subnational regions. The proxy was satellite data on lights at night.

Big Data may lead to knowledge enhancement, but on the other hand not all data belonging to Big Data are reliable. Part of Big Data are the so-called dirty data, including fake data. For knowledge acquisition it is important to detect possible dirty data, including fake information.

\footnotetext{
${ }^{10}$ Henderson, Storeygard, Weil (2009).
} 


\section{Machine learning}

The mentioned Big Data are to be analysed. Since they are large, changing and heterogeneous, they require appropriate methods of analysis. This has been more feasible not only because of 'hard' innovations, but predominantly because of the application of more or less advanced methods of data analysis.

Machine learning is sometimes considered to be part of so-called Artificial Intelligence, which is understood as computer systems that are capable of performing tasks that previously required human intelligence. Machine learning is often understood as computer programs that can 'learn' how to complete tasks and improve their performance over time.

In my opinion, machine learning is nothing other than a set of algorithms based on the methods that can be considered as exploratory (rather than confirmatory) statistical methods. In fact, very many of these methods have been known for many years. With the dynamic development of computer technology, they can now be used for very large data sets and the results can be obtained in a short time.

There are four basic groups of machine learning methods, in which the studied objects are described by data.

Discrimination (classification, supervised learning) methods involve determining and discriminating functions for the objects whose assignment into classes is known. Then new observations can be easily assigned to these known classes.

Classification (clustering, unsupervised learning) methods involve classifying objects into unknown classes, in such a way that the objects belonging to the same class are as 'similar' as possible, while the objects belonging to different classes are as 'dissimilar' as possible.

Dimensionality reduction methods involve transforming many variables describing objects into a small number of dimensions, for example 2 or 3 , to allow visualization, leading to better interpretation.

Regression methods involve the determining function which reflects the dependence of one variable on some other variables.

\section{Distributed ledger technology}

Distributed ledger technology (DLT) is the infrastructure that is most well-known in its blockchain version. This technology allows simultaneous access, validation and reporting across a network that is spread across numerous members of a decentralized network. The important feature of DLT is that the network is distributed rather than centralized. This means that there is no central party which takes control over the network.

The concept of DLT was proposed by Haber and Stornetta. ${ }^{11}$ In a paper published by the Journal of Cryptology, they proposed a solution in which a document uploaded to a network gets stamp, which identifies the author

\footnotetext{
11 Haber, Stornetta (1991).
} 
and time of the document. This information is encrypted, therefore it cannot be changed. This concept was then popularized by Bitcoin and now is referred to as blockchain.

DLT can be treated as kind of database or spreadsheet. Its entries are recorded, stored, and distributed across a network of participants so that each participant has a matching copy of the digital database. The technology is transparent, the parties conducting the transaction are identified in a given moment. Blockchain can be used not only for reporting transactions but also for arranging contracts between two parties. The main feature of blockchain is the almost real time reporting, therefore it can revolutionize, for example, financial reporting.

The above-mentioned technological changes are studied by the scientists of different disciplines. This also includes research in the economic sciences. In my opinion, one possible stream is the contribution of technological innovation to economic growth through the endogenous growth model. ${ }^{12}$

\section{TECHNOLOGY IN THE ECONOMIC SCIENCES: THE SECOND CONTEMPORARY TREND}

The second contemporary trend which has had (and will continue to have) an impact on research in the economic sciences is related to the involvement of technology, particularly the elements that were presented in the previous section.

In my opinion, this impact can be classified as twofold:

- the methodological changes in the research,

- the organizational changes in the research.

The methodological changes in the research are driven by two elements presented in the previous section, namely access to Big Data and the use of machine learning algorithms.

Possible access to Big Data gives scientists the possibility to conduct research based on numerous heterogeneous data sets, many more than those that were used in the past. The traditional data used in economic research were mostly numerical data, gathered either from primary sources (acquired by a survey method) or from secondary sources (public statistics, reports from companies). In some cases, textual data have also been used.

In addition, machine learning methods helps scientists to explore data to acquire knowledge, which may become the basis for new insights and the building blocks of a new theory.

This leads to the increasing pressure to use quantitative methods, both confirmatory and exploratory ones, since there is almost unlimited access to these methods. The main problem is now to get significant results.

Another impact of technology on scientific research has been observed in the organization of scientific research; it can be considered as an organizational framework. The main characteristics of this framework are as follows:

12 Romer (1990). 
1. Researchers have practically unlimited access to databases of publications containing the results of relevant research, in addition they can use search engines to systematize the available results.

2. Researchers have very fast access to the newest scientific results. They can be immediately alerted about either the publication of these results or just the availability of draft results on a website.

3 . There is almost an unlimited opportunity for teams from different universities located in different parts of the world to conduct research together. This is achieved by online communication and the use of cloud computing for parallel elaboration of the parts of the research.

4. There are a lot of supporting online tools that can be used for the effective dissemination of results, including automated proof-reading systems.

The developments in scientific research resulting from technological innovation lead to the question: to what extent could scientific research evolve in the same direction as typical business. One of the key changes in business resulting from technological development is the automation of jobs. Not only manual work is being replaced by robots, but also human brain processes are being replaced by computer programs. In my opinion, the jobs which will be not automated in the short term have three features:

- they require creativity, so the jobs involving repetitive work are (and will be) automated,

- they are customized (tailored to the needs of the individual customer),

- they require the mobility and flexibility of the employee.

Therefore, we may be interested in whether automation can influence scientific research and to what extent computer programs (automated machines) can participate in conducting scientific research.

One of the very first examples of a so-called robot scientist was 'robot Adam', which in 2004 conducted independent experiments and interpreted the results of these experiments in drug research. ${ }^{13}$

In my opinion, there are pros and cons as far as automation of research is concerned.

In terms of the advantages that automated machines have over humans, one must mention:

- more precise logical inference,

- analysis using Big Data and machine learning,

- acquisition of information from thousands of scientific publications,

- lower costs of research, given scalable automated systems,

- a shorter process to develop automated machines, when compared with the periods required to educate scientists,

- effective use ( 24 hours a day, 7 days a week).

As we can see, most of these advantages are in the area of cost and effectiveness. Also, these advantages are applicable in the case of highly developed systems which are also transparent for end users - scientific teams. Similarly, as with other types of activities, replacing humans is possible only for

${ }^{13}$ King et al. (2004). 
repeatable parts of the research. Examples of repeatable types of research are conducting a very large number of similar tests (even statistical tests) and interpreting the results with some standard scheme.

Of course, there are also some disadvantages associated with the automation of scientific research. The main negative feature is limiting the role of humans in scientific research which - in the case of very advanced computer systems - will lead to the threat for the profession of researcher. However, in my opinion, the automation of research in economic sciences is limited to the acquiring and processing large data sets through machine learning algorithms. The main role of the human researcher will be formulating hypotheses, designing research, and drawing conclusions for theory and for practitioners.

Calabresi, G. (1961). Some thoughts on risk distribution and the law of tarts. Yale Law Journal 70(4): 499-553.

Coase, R. (1961). The problem of social cost. The Journal of Law and Economics 3(1): 1-44.

Granovetter, M. (1985). Economic action and social structure: the problem of embeddedness. American Journal of Sociology 91(3): 481-510.

Guiso, L., Sapienza, P., Zingales, L. (2004). The role of social capital in financial development. American Economic Review 94(3): 526-556.

Haber, S., Stornetta, W.S. (1991). How to timestamp a digital document. Journal of Cryptology 3: 99-111.

Henderson, J.V., Storeygard, A., Weil, D.N. (2009). Measuring Economic Growth from Outer Space. NBER Working Paper No. 15199.

Jajuga, K. (2019). Nauki ekonomiczne - dylematy klasyfikacji dyscyplin. Tendencje zmian, [in:] Ewolucja nauk ekonomicznych: 140-150. Warsaw: PAN.

Kahneman, D., Tversky, A. (1979). Prospect theory: an analysis of decision under risk. Econometrica $47(2): 263-292$.

King, R.D., Whelan, K.E., Jones, F.M., Reiser, P.G.K., Bryant, C.H., Muggleton, S.H., Kell, D.B., Oliver, S.G. (2004). Functional genomic hypothesis generation and experimentation by a robot scientist. Nature 427(6971): 247-252.

Lindbeck, A. (2008). The Sveriges Riksbank Prize in Economic Sciences in Memory of Alfred Nobel 1969-2007. <www.nobelprize.org>.

OECD Classification. <http://www.oecd.org/science/inno/38235147.pdf>.

Platt, M.L., Glimcher, P.W. (1999). Neural correlates of decision variables in parietal cortex. Nature 400(6741): 233-238.

Romer, P.M. (1990). Endogenous technological change. Journal of Political Economy 98(5): 71-102.

\section{THE DEVELOPMENT OF THE ECONOMIC SCIENCES: TWO CONTEMPORARY TENDENCIES}

Sum mary

The paper discusses two important trends that have shaped research in the economic sciences over the last two decades. The first one is the growing interdisciplinarity of economic research. The second one is the impact of technological changes on research in the economic sciences. The paper presents the author's systematization of these problems.

Keywords: economic sciences; interdisciplinarity; technology; JEL Codes: A1 